\title{
Physics of Resistive Wall Modes
}

\author{
V. Igochine \\ MPI für Plasmaphysik, Euratom-Association, D-85748 Garching, Germany
}

Email: valentine.igochine@ipp.mpg.de

\begin{abstract}
The advanced tokamak regime is a promising candidate for steady state tokamak operation which is desirable for a fusion reactor. This regime is characterized by a high bootstrap current fraction and a flat or reversed safety factor profile, which leads to operation close to the pressure limit. At this limit, an external kink mode becomes unstable. This external kink is converted into the slowly growing Resistive Wall Mode (RWM) by the presence of a conducting wall. Reduction of the growth rate allows one to act on the mode and to stabilize it. There are two main factors which determine the stability of the RWM. The first factor comes from external magnetic perturbations (error fields, resistive wall, feedback coils, etc). This part of RWM physics is the same for tokamaks and reverse field pinch (RFP) configurations. The physics of this interaction is relatively well understood and based on classical electrodynamics. The second ingredient of RWM physics is the interaction of the mode with plasma flow and fast particles. These interactions are particularly important for tokamaks, which have higher plasma flow and stronger trapped particle effects. The influence of the fast particles will also be increasingly more important in ITER and DEMO which will have a large fraction of fusion born alpha particles. These interactions have kinetic origins which make the computations challenging since not only particles influence the mode, but also the mode acts on the particles. Correct prediction of the "plasma-RWM" interaction is an important ingredient which has to be combined with external fields influence (resistive wall, error fields and feedback) to make reliable predictions for RWM behavior in tokamaks. All these issues are reviewed in this paper.
\end{abstract}




\section{Introduction}

The main motivation for fusion research is the development of a reliable and virtually inexhaustible energy source. From the engineering point of view it is attractive to have a stationary solution for this problem. The most advanced present day concept for plasma confinement is the tokamak. Thus, steady state tokamak operation could be an attractive regime for a future fusion power plant based on the tokamak concept. Constant power production in this regime is more convenient both for energy conversion and for consumers. At the same time, constant power loads on the plasma facing component allow more simple power handling, lower engineering efforts for reactor design, and smaller costs for the fusion power plant. A necessary requirement for tokamak operations is a constant plasma current, which produces the poloidal component of the magnetic field. This component twists magnetic field lines which ensure plasma confinement in tokamaks. A non-inductive source for the constant plasma current is required for steady state operation because an inductive drive can work only temporarily. There are several different options for such non-inductive current drive in tokamaks: Electron Cyclotron Current Drive (ECCD), Lower Hybrid Current Drive (LHCD), Neutral Beam Current Drive (NBCD) and bootstrap current [1,2]. Among them only the bootstrap current can produce a sufficiently large amount of the toroidal current in big tokamaks. All other options could be very useful for plasma control, but will play a negligible role in the total toroidal current balance during the flattop phase of a discharge. Thus, all modern concepts for steady state DEMO reactors rely completely on the bootstrap current as the main current source in the plasma. The amount of bootstrap current is proportional to the pressure gradient [2]. Typically the maximal pressure gradients are located off-axis which leads to flat or hollow current profiles in the plasma. In this case, the minimum safety factor value is about two, which avoids some dangerous resonant surfaces $((1,1)$ kink mode and $(3,2)$ Neoclassical Tearing Mode). For even higher safety factor values the most dangerous $(2,1)$ resonant surface could also be avoided. This is a positive consequence of the elevated safety factor profile in the advanced tokamak scenario. At the same time, high bootstrap current fraction can only be achieved with high pressure gradients (which are unavoidable for advanced tokamak scenarios). It is well known from MHD that current and pressure gradients lead to instabilities. In advanced tokamak scenarios the high pressure gradients result in external kink instabilities. In the presence of a resistive wall the kink 
instability is converted into the more slowly growing Resistive Wall Mode (RWM). This instability is the main topic of the present paper. The control of this instability was also the subject of a very comprehensive review by Chu and Okabayashi [3] two years ago. Here, we attempt to present a concise review of resistive wall mode physics, focusing on the mechanisms behind the mode behavior and only slightly touching on the control issues described in detail in Ref [3]. We start from the basic physics, focus on the recent achievements and discuss possible future steps.

The paper consists of eight sections. In section 2, the main stability limits are defined and discussed. We recall some basic information about the simple dispersion relation for RWM and its consequences in section 3. The RWM structure is discussed in section 4. Section 5 is devoted to the physics of the resistive wall mode and its interaction with plasma and external magnetic perturbations (error fields, resistive wall, feedback coils, etc.). Predictions for ITER are discussed in section 6. Triggering of the RWM is discussed in section 7. Finally, in section 8 we summarize the main results and outline guidelines for future studies of RWMs.

\section{Stability boundary of the resistive wall mode}

As mentioned before, the resistive wall mode is a pressure gradient driven instability. A typical characteristic of the total pressure in a tokamak is the normalized plasma pressure $\beta=2 \mu_{0}\langle p\rangle / B_{t}^{2}, \beta_{N}=\beta\left(a B_{t} / I_{p}\right)\left(\langle p\rangle\right.$ is the average plasma pressure, $B_{t}$ is the toroidal magnetic field, $a$ is the minor radius and $I_{p}$ is the plasma current). Using this quantity, it is possible to define two limits for a given external kink mode. Assuming a plasma without an external wall, one obtains an external kink by increasing $\beta_{N}$ above a particular threshold value called the "no wall" $\operatorname{limit}\left(\beta_{N, \text { no-wall }}\right)$. On the other hand, if a perfectly conducting wall is considered instead of a real wall, one could increase $\beta_{N}$ further and reach the instability threshold at a considerably higher value, which is called the "ideal wall" limit $\left(\beta_{N, \text { ideal-wall }}\right)$. In either cases, the external kink grows extremely fast after crossing a threshold value (growth rate: $\gamma_{0} \sim 1 / \tau_{\text {Alfven }}, \tau_{\text {Alfven }}=L / v_{\text {Alfven }}, v_{\text {Alfven }}=B_{t} / \sqrt{\mu_{0} \rho}, L$ is the characteristic length and $\rho$ is the plasma density). At this point, the control of the mode becomes impossible and the confinement is lost. The situation changes if one considers a real resistive wall. In this case the external kink is still stable before the "no wall" limit $\left(\beta_{N}<\beta_{N, n o-\text { wall }}\right)$ and highly unstable 
above the "ideal wall" limit as before $\left(\beta_{N}>\beta_{N, \text { ideal-wall }}\right)$, but in the range between the "no wall" and the "ideal wall" limits $\left(\beta_{N, \text { no-wall }}<\beta_{N}<\beta_{N, \text { ideal-wall }}\right)$ the mode splits into two branches [4]:

- The fast branch, which is the normal external kink mode, is stable in this region because the rotation of this mode is relatively fast with respect to the wall. At these frequencies the resistive wall acts as an ideal wall and stabilizes this branch.

- The slow branch on the other hand has a lower rotation frequency, which enables the mode perturbation to penetrate through the wall and allows this branch to become unstable. But, the growth rate of the mode becomes much smaller than the inverse Alvfen time (growth rate: $\gamma_{0} \sim 1 / \tau_{\text {wall }}$, $\tau_{\text {wall }} \approx \tau_{d} / 2 m, \tau_{d}=\mu_{0} b d / \eta_{\text {wall }}$, where $b, d$, and $\eta_{\text {wall }}$ are the wall radius, thickness, and resistivity, and $m$ is the dominant poloidal mode number). Here, the wall properties become important because they determine the flux penetration. At such slow time scales control of the mode becomes possible.

In summary, the question of resistive wall mode stabilization in tokamaks is a question of gaining the region of the operation space between the "no-wall" and "ideal wall" limits. For advanced operations close to the pressure limits the ratio between these two limits is about $\beta_{N, \text { ideal-wall }} / \beta_{N, n o-\text { wall }} \sim 1.5-2$, which when translated to fusion power is an increase by a factor of 3-4 in the case of RWM stabilization (fusion power scales approximately with $\beta_{N}^{2}$ ). This factor is a crucial component which could make the advanced scenario comparable to conventional H-mode discharges in terms of fusion power. In this regime the bootstrap current fraction is close to one $\left(f_{b j}=J_{\text {bootstrap }} / J_{\text {plasma }} \approx 1\right)$. Thus, stabilization of the resistive wall mode and operation close to the "ideal wall" limit are extremely important. One has to note that in the conventional scenario this gap between the "no-wall" and "ideal wall" limits is small and gaining this region just slightly increases the operational space without strong benefits in fusion performance. Comparisons of the "ideal wall" limit and the "no wall" limit are shown for two different equilibriums in figure 1 [5]. The gap between the limits depends strongly on the toroidal mode number and safety factor profile. For most unstable $n=1$ modes one has a substantial increase in $\beta_{N}$ with a reversed shear q-profile and only a moderate increase in the case of a monotonic safety factor profile. Therefore, RWM stabilization is only 
really worthwhile, in terms of performance gain, in advanced tokamak operation scenarios, which feature reversed or near reversed shear q-profiles.
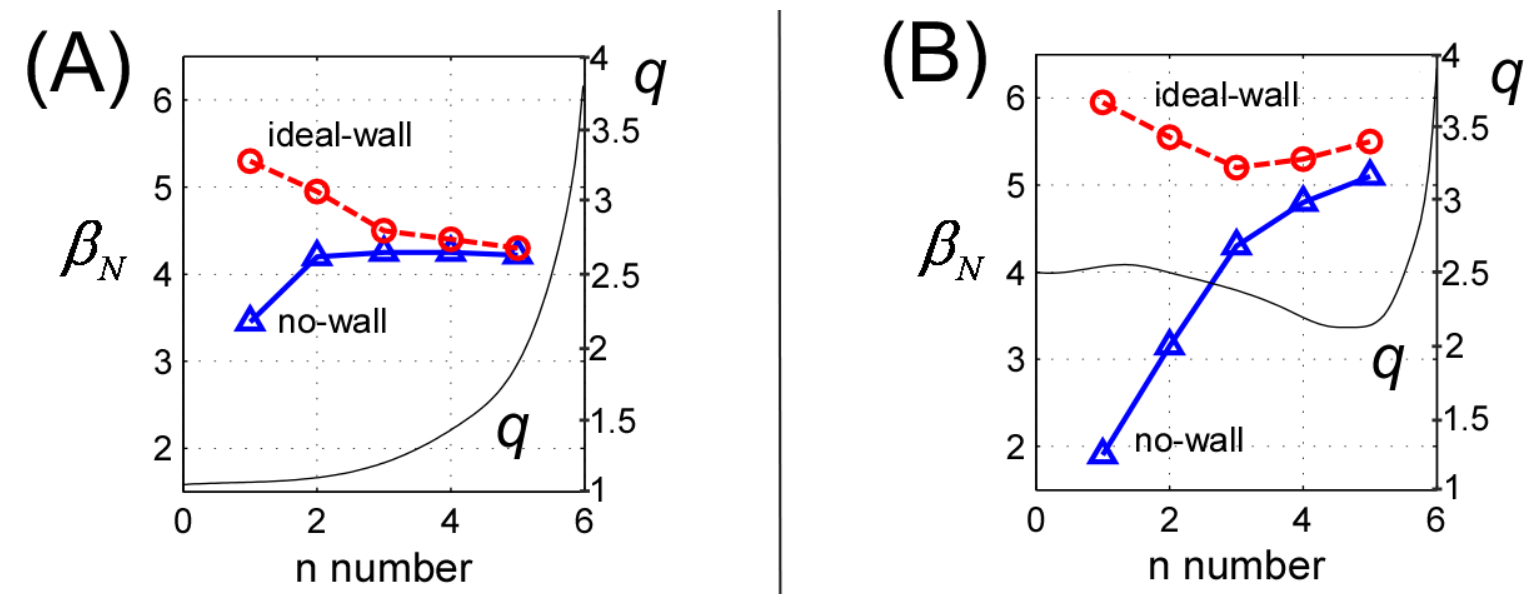

Figure 1. The "ideal wall" and the "no wall" limits are shown for different toroidal mode numbers. (A) The case of the monotonic safety factor profile is considered. (B) The reversed shear safety factor profile is considered. The figures are adopted from ref.[5]

There is also another measure for the plasma stability with respect to the resistive wall mode which is used in some papers and should be mentioned. One can define the internal inductance, which is a relation between the averaged poloidal magnetic field and its value at the plasma boundary, $l_{i}=\left\langle B_{\theta}^{2}\right\rangle / B_{\theta a}^{2}$. This is an integral measure of the peakedness of the current profile for a particular equilibrium. The internal inductance can be used to estimate the "no wall" $\left(\beta_{N, \text { no-wall }}\right)$ and "ideal wall" $\left(\beta_{N, \text { ideal-wall }}\right)$ limits, but the relations are not universal. For example, a typical relation for the "no wall" limit in the DIII-D tokamak could be either $\beta_{N, \text { no-wall }} \approx 4 l_{i}[6]$ or $\beta_{N, n o-\text { wall }} \approx 2.4 l_{i}$ [7] depending on the discharge scenario. For this reason we use the quantity $\beta_{N}$ as the measure for the plasma stability against the resistive wall modes. These stability limits in terms of $\beta_{N}$ depend strongly on the plasma profiles, plasma shape, propertires of the resistive wall and the aspect ratio. The strong aspect ratio dependence can be seen, for example, in a comparison of NSTX (aspect ratio=1.27) and DIII$\mathrm{D}$ (aspect ratio=3.1) discharges with similar $l_{i}$ values. The $\beta_{N, n o-\text { wall }}$ and $\beta_{N, \text { ideal-wall }}$ values are a factor of two larger for the lower aspect ratio case (NSTX) compared to the high aspect ratio case (DIII-D)[8]. Changes in the plasma shape can also influence these limits even if $l_{i}$ is the same. Thus, all these ingredients have to be taken correctly into account to calculate the "no wall" and the "ideal wall" limits using linear MHD codes. Unfortunately, even this (complex) determination of the plasma stability boundaries does not give a final answer for 
the boundaries of RWM unstable region. The actual plasma stability is a very complex subject which depends also on kinetic effects and can not be described only in terms of $\beta_{N}$, as will be show below.

\section{Simple dispersion relation for the resistive wall mode}

In the previous section, the RWM stability boundaries were defined. In order to understand the behavior of the instability inside of the boundaries, a simple dispersion relation is discussed here. A relatively simple form of such a dispersion relation was derived from a set of linear MHD equations [9,10]. This simplest form has no kinetic effects and assumes a fluid plasma viscosity:

$$
\underbrace{\delta W_{p}}_{\substack{\text { ideal } \\
\text { MHD }}}+\underbrace{\left(\gamma+i n \Omega_{0, p l}\right) D}_{\begin{array}{c}
\text { plasma } \\
\text { rotation }
\end{array}}+\underbrace{\frac{\delta W_{v}^{b} \gamma \tau_{w}+\delta W_{v}^{\infty}}{\gamma \tau_{w}+1}}_{\begin{array}{c}
\text { resistive } \\
\text { wall }
\end{array}}=0
$$

Here $\Omega_{0, p l}$ is the rotation frequency of the plasma, $\left(\gamma+i n \Omega_{0, p l}\right) D$ is the energy dissipated in the plasma, $\delta W_{v}^{b}$ and $\delta W_{v}^{\infty}$ are perturbed energies in vacuum with an ideal wall at $b$ and at $\infty$, respectively, $\tau_{w}$ is the wall time, $\gamma=\gamma_{0}+i \omega_{R W M}$ is the complex growth rate of the mode, and $n$ is the toroidal mode number. The dispersion relation contains parts related to the ideal stability of the mode, the influence of the plasma rotation (interaction of the mode and the plasma) and the influence of the resistive wall. The important consequences of the dispersion relation are shown schematically in figure 2 . The plasma without a wall can be confined up to the "no wall" limit after which a fast external kink instability develops (magenta line). In the presence of an "ideal wall" this limit is shifted to higher $\beta_{N}$ values (up to $\beta_{N, \text { ideal-wall }}$ ). In both cases, the control of an ideal instability above these limits is not possible. In the real situation, between the "no wall" and "ideal wall" limits a slowly growing resistive wall mode develops. The resistive wall prevents a fast penetration of the flux from the mode and determines the growth rate of the mode. Applying the feedback controlled external currents one reduces the flux penetration further and suppresses the mode. This type of stabilization opens up a stability window above the "no wall" limit, as shown in figure 2 . The second important effect is the plasma rotation. In the presence of a viscosity/dissipation, the plasma drags the mode. As a result of such a "drag force", the mode rotates faster and becomes decoupled from the wall, which prevents penetration of the flux through the wall and again stabilizes the mode. The coupling between the plasma and the mode ("drag force") is stronger for high $\beta_{N}$ values 
because of the higher dissipation at higher $\beta_{N}$. This effectively opens a stability window from the "ideal wall" limit if the rotation stabilization is dominant ${ }^{1}$.

In summary, the RWM growth requires flux penetration through the wall. Two different mechanisms can prevent, reduce or suppress this penetration: active feedback control which is more effective close to the "no wall" limit, or from "ideal wall" limit stabilization via plasma rotation.

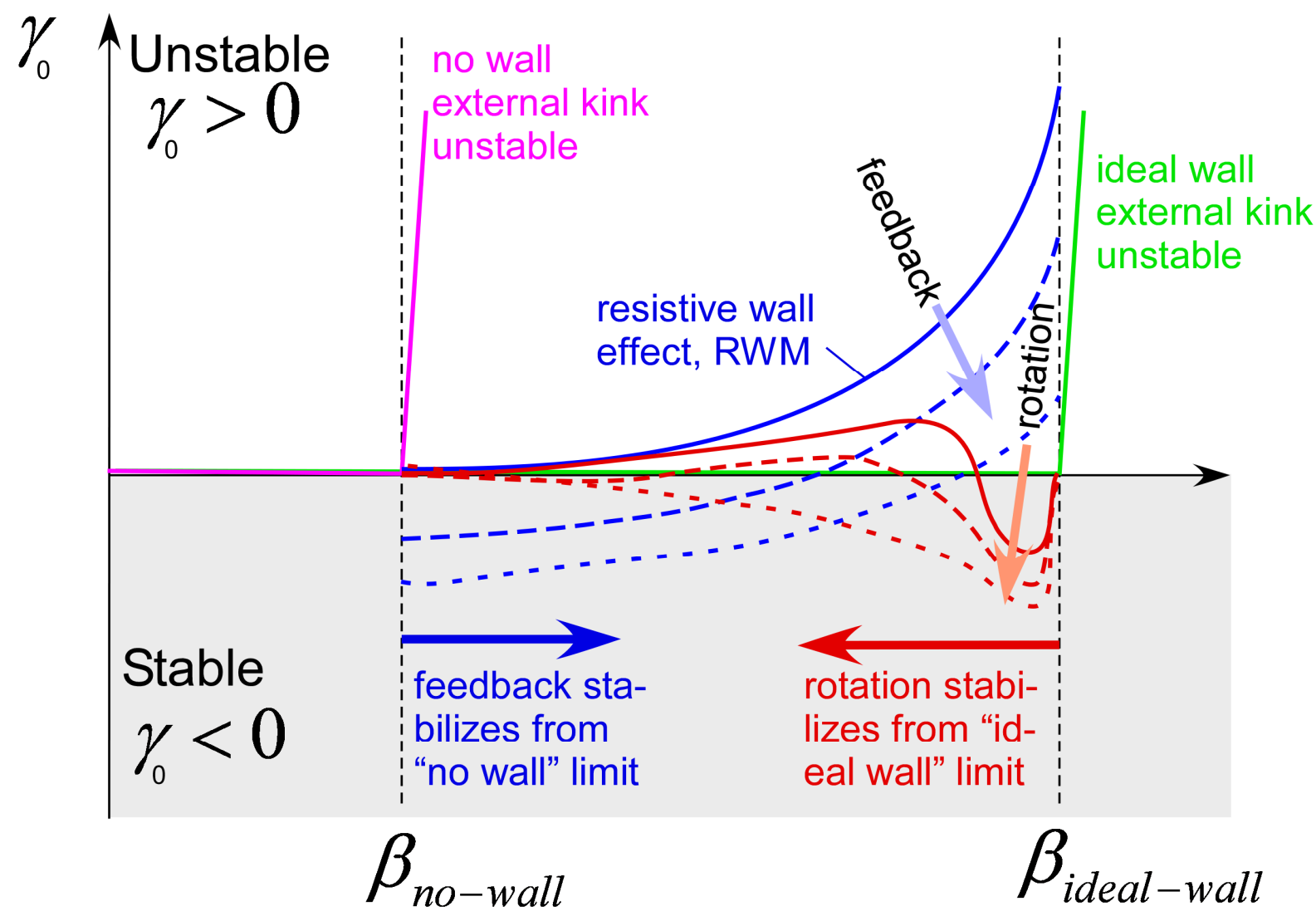

Figure 2. (color online) Schematic representation of the RWM behavior between "no wall" and "ideal wall" limits from the dispersion relation (eq.1). The mode is unstable above the "no wall" if no wall is present (magenta line). The mode is stable up to $\beta_{\text {ideal-wall }}$ in case of an ideal wall (green line). Slowly growing mode is unstable between the "no wall" and "ideal wall" limits (blue curves). Active feedback pushes this curve down and stabilizes from "no wall" limit. Inclusion of the plasma rotation stabilizes the RWM from the "ideal wall" limit (red curves).

\footnotetext{
${ }^{1}$ The presented figure 2 gives only schematic description of RWM behavior. It contains some simplifications to make the figure more clear. For example, growth rates values, $\gamma_{0}$, for "no wall external kink" and "ideal wall external kink" cases are set to zero in the stable region to reduce the number of curves. These values could be strongly negative in the reality.
} 


\section{Structure of the resistive wall mode}

Experiments show that both the internal factors (plasma rotation, etc) and external factors (resistive wall, external coils, etc) have a strong impact on the mode stability [11-14]. A physical basis for these strong effects is a broad eigenfunction of the mode. Resistive wall modes typically have a toroidal mode number $n=1$ and multiple poloidal mode numbers $m=2 \ldots 7$ which are coupled together. There are also situations in which the higher toroidal mode numbers $(n \geq 2)$ can be excited simultaneously. These multiple unstable eigenvalues can coexist together in the plasma and were observed for example in high beta NSTX plasmas [15].

It is clear that the external kink part of the RWM eigenfunction creates currents at the plasma boundary. These currents are close to the wall and lead to strong interactions with the currents in the wall and with externally produced perturbations. The influence of the internal currents, located around mid-radius of the plasma, on this interaction is smaller due to the larger distance to the wall. At the same time, the internal part of the eigenfunction is responsible for the strong interaction with the plasma flow. This interaction has a kinetic origin which results in interaction of the wave (mode perturbations due to RWM) with particles, as will be discussed later. The external kink part of the eigenfunction is less important for the interaction with the plasma, since the amount of plasma particles in the core is much higher compared to the plasma boundary region. This conclusion is also confirmed by the kinetic calculations, as will be shown later. Typical displacement eigenfunctions of the resistive wall mode for two slightly different edge safety factors in DIII-D are shown in figure 3 [16]. The internal part is non-negligible even for cases in which the external kink is dominant. Calculations for JET indicate that the amplitude of the internal displacement is of the same or even significantly larger than the external kink part [17]. Actually, only a global character which has internal and external parts of the RWM eigenfunction can explain simultaneous effects of the resistive wall, external perturbations and plasma rotation, as observed experimentally. 

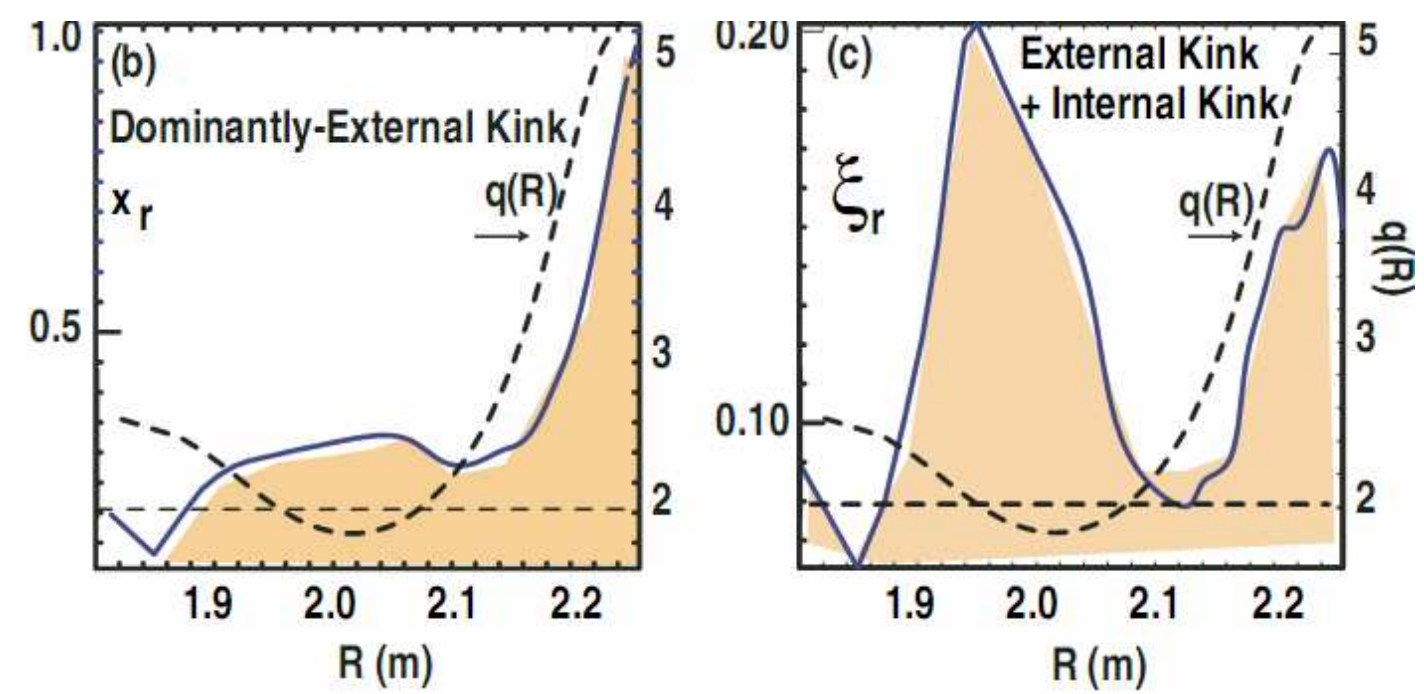

Figure 3. Shape of the radial displacement eigenfuction for two slightly different edge safety factors (left $q_{a}=5.6$; right $q_{a}=6$ ). The figures are adopted from Ref. [16]. The internal part is present in both cases but has much stronger relative amplitude on the right. These figures result from linear MHD calculations which imply that only shapes of the eigenfunctions could be compared. (A non-linear MHD simulations are necessary for the absolute values of the displacements.)

The last important issue concerning the mode structure is the mode "rigidity". It characterizes the rigidity of the mode structure to external actions. This term is defined differently by different authors. Typically one of the following definitions of the mode "rigidity" is used:

1) The mode "rigidity" in real space assumes that local poloidal (and/or toroidal) suppression of the RWM in one place leads to overall reduction of the mode amplitude.[18]

2) The mode number "rigidity" assumes that suppression of the most unstable toroidal mode number $(\mathrm{n}=1)$ would not destabilize a mode with higher $\mathrm{n}$-numbers. [19,20]

3) For the "perturbative" calculations discussed in the following sections mode "rigidity" means that the shape of the eigenfunction is fixed in the analysis of the particle mode interaction using a kinetic approach (see section 5.2).

These definitions are not independent. For example, a non-rigid case in terms of the first definition could give different spectra and lead to a non-rigidity in terms of the second definition. One has to be careful to understand the exact meaning of the word "rigidity" in a particular case. In many cases, people assume a "rigid" mode structure of RWM using one of these definitions.

\section{Stability of the resistive wall mode}

The physics of the resistive wall mode is quite complicated and is not yet completely 
understood. Different factors like plasma rotation, fast particles, resistive walls, error fields and feedback impact the mode stability and could be dominant under different circumstances. Thus, resulting stability/instability of RWM is an integral quantity which contains all of these factors. Change in even one of the ingredients could completely change the picture and drive the instability from a stable to an unstable region or vice versa. Some of these factors are well understood, but others still require detailed investigations.

\section{Resistive Wall Mode}

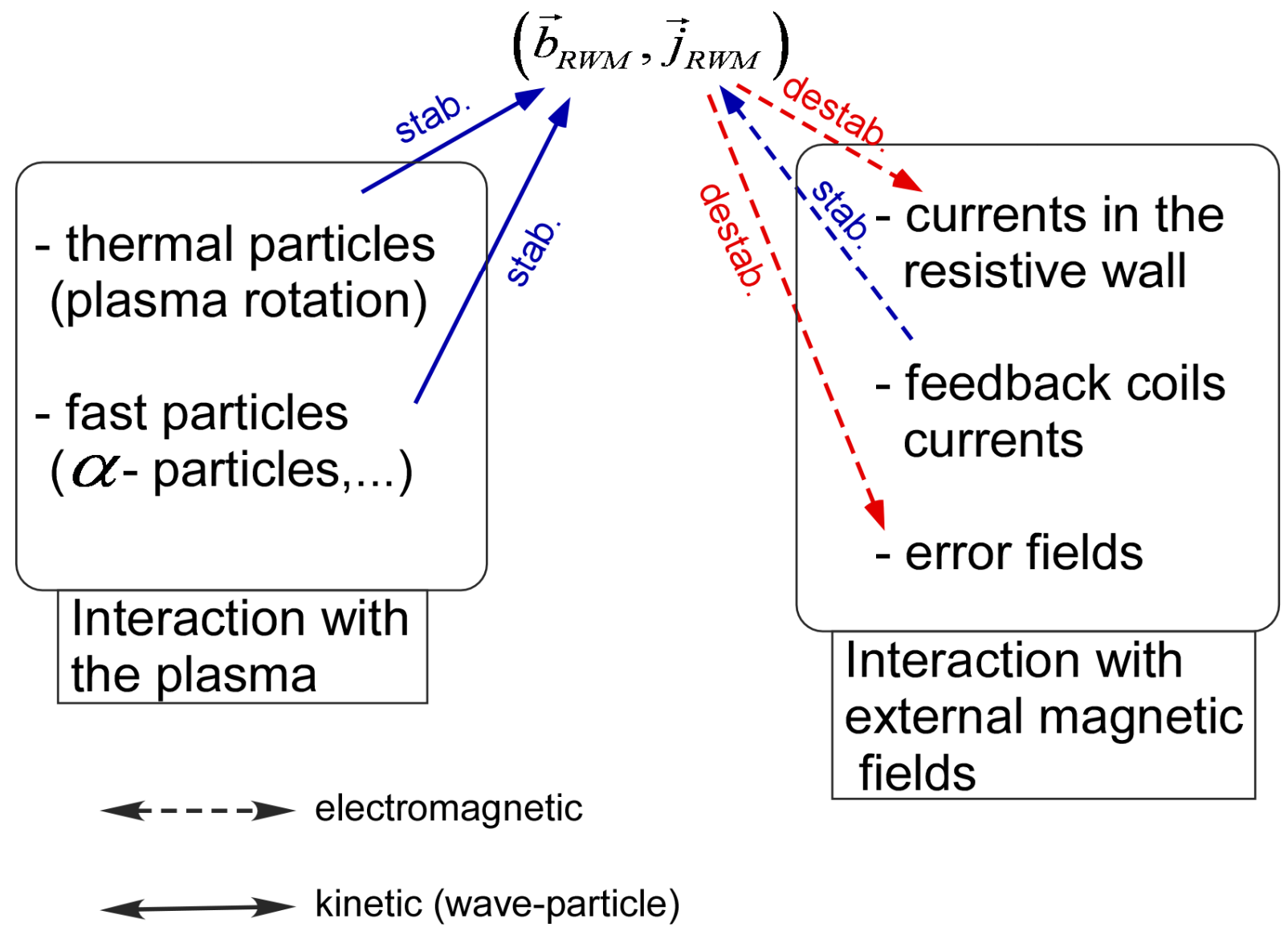

Figure 4. (color online) Schematic representation of the Resistive Wall Mode interactions with the plasma and external magnetic fields. Different effects are separated in two groups depending on the type of interaction. Typical stabilizing and destabilizing influences are marked. The amount of stabilization/destabilization depends strongly on the particular situation (profiles, plasma shape, particles distribution, etc.).

It is useful to separate all these factors into two groups depending on the physics background (see figure 4). This allows us to gain a better physical understanding of RWM physics:

(1) In the first group we include all of the effects which could be described purely by electromagnetism. These are the interaction of the mode with any external magnetic fields which could originate from the resistive wall, error fields or feedback coils. In this case only perturbation currents due to the mode inside the plasma enter into the problem. Actually, the 
situation could be even more simplified since these currents could be substituted by an appropriate choice of the surface current on the plasma boundary (or boundary conditions at the last closed flux surface in the calculations). The solution of such a problem in real geometry would be complex, but the approach is straightforward. This part of the physics is discussed in section 5.1 .

(2)The second group contains the interaction of the RWM with the plasma. This means the influence of the plasma rotation and of fast particles on the mode stability. These effects have kinetic origins and have to be considered as a wave-particle interaction. Self-consistent calculation of the mode within kinetic approach is an extremely challenging task, as will be shown later. Thus, an assumption on this interaction which could then be used in a fluid approach within an MHD code is required. In spite of the significant progress during last few years, this piece of physics is still not complete. This will be discussed in detail in section 5.2.

\subsection{RWM interaction with external magnetic fields}

Many simple models for resistive wall mode stability were established at the beginning of the RWM research to investigate the interaction of the RWM with the wall and with feedback currents. The most widely used models were proposed by V.D.Pustovitov[21],

A.H.Boozer[22], M.Okabayashi[23], J. M.Finn[24] and R. Fitzpatrick [25]. In the following, the Fitzpatrick model [25] is discussed. This model is a good illustration which shows a typical simple model approach and its limitations. Schematic representation of the Fitzpatrick model is shown in figure 5. As was mentioned before, all currents due to the RWM inside of the plasma volume can be substituted by a single surface current (boundary conditions). This approach is implemented in the cylindrical Fitzpatrick model in the most straightforward manner. The resistive wall mode is represented by the outer surface current layer. The inner most region is filled by ideal plasma. An inertial layer between the plasma and the current layer (RWM) serves to match the difference in rotation between the plasma and the resistive wall mode, because in a typical situation the plasma rotates much faster. These assumptions are sufficient to derive a dispersion relation for the resistive wall mode. Using this dispersion relation, and assuming a particular viscosity and dissipation, one can investigate the behavior of the mode for different plasma rotations. 


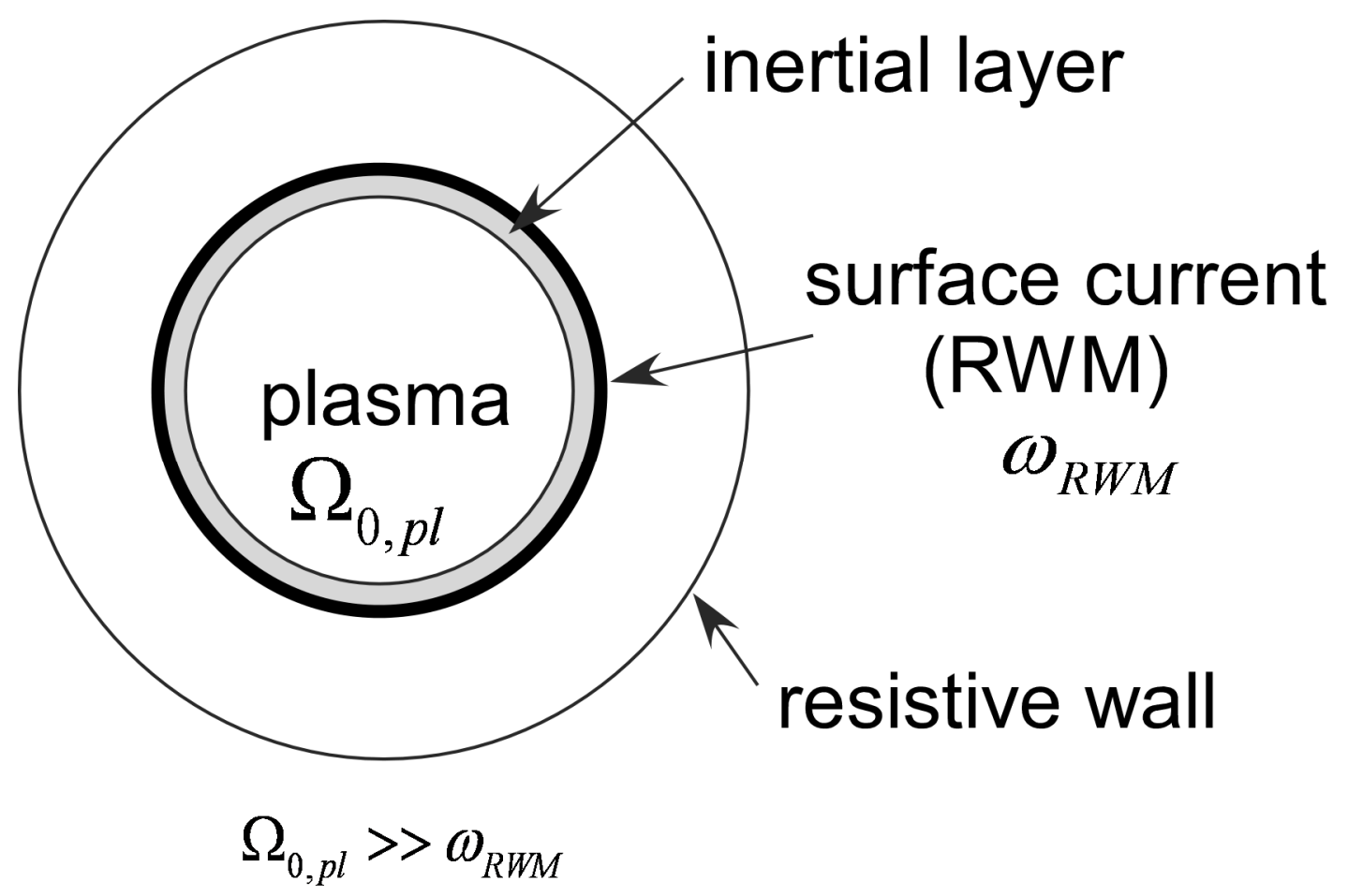

Figure 5. Schematic representation of the cylindrical Fitzpatrick model [25]. The RWM is represented by an external current layer which is connected to the ideal plasma region via an inertial layer. This layer is $n$ eeded to match slow rotations of the RWM $\left(\omega_{R W M}\right)$ and fast rotation of the plasma $\left(\Omega_{0, p l}\right)$.

In these models kinetic effects are described by one or two free parameters (representing viscosity and dissipation in the Fitzpatrick model, only dissipation in the Boozer model etc.). This oversimplification of plasma-mode interaction is the main problem of all simple models. One can determine these free numbers for a given experiment, but scaling to other tokamaks is impossible (or even scaling to substantially different regimes in the same tokamak). At the same time, these simple approaches are extremely useful for getting a basic understanding of RWM physics. They also become powerful tools in the case of weak interaction between the resistive wall mode and the plasma (when kinetic effects are small). Such a situation arises in reversed filed pinches (RFPs), which also have resistive wall modes.

Resistive wall mode physics in RFPs is not identical to that in tokamaks. The RWM in RFPs is a current driven instability in contrast to the pressure driven RWM in tokamaks, which is a primary concern in advanced scenario. In addition, the interaction between the plasma and the mode play an important role in tokamaks and is negligible in RFPs [26, 27]. This difference actually makes RFPs a good test device for the electromagnetic part of RWM physics. Additional advantages for these tests come from the large number of control coils which completely cover the surface of the RFP device. In the tokamak case, only a high field side set of coils is typically installed. One has to note that the current driven RWMs are also investigated in the tokamaks utilizing their high reproducibility to study the RWM control 
issues. The structure of the current driven RWM at the plasma surface is similar to what is expected for pressure driven RWMs and thus could be used for investigation of the RWM interaction with external currents and walls [28]. At the same time the pressure driven RWM could have quite a different eigenfunction in the plasma core with higher amplitudes of the internal components and a much richer poloidal spectrum [29]. Thus, the plasma-RWM interaction could be quite different for current driven and pressure driven RWMs in tokamaks.

During the last few years, a significant progress in the field of RWM physics was achieved in RFPs. This reflects a good understanding of the RWM interaction with external magnetic fields. There are several points which are particularly interesting in the obtained results:

- Simultaneous stabilization of several RWMs was achieved for the first time in T2R and confirmed in other devices [30, 31, 32].

- The unlocking and controllable rotation of RWM using feedback coils were demonstrated for the first time in RFX-mode and confirmed in other experiments $[33,34,35,36]$.

- The real vessel structure was taken into account in the RWM stability calculations and was shown to affect the growth rates of the RWM instability[37].

- It was shown that simple RWM models could be used to calculate some quantities rather accurately, for example, for the experimentally measured frequency of RWM rotation experiments agrees very well with theoretical predictions [35, 38].

All these results show that the electromagnetic part of the resistive wall mode physics is relatively well understood. Application of this knowledge to real tokamaks is a complex but solvable problem. This is shown by the implementation of the real wall in different codes [39, 40]. Real 3D geometry of the wall is important not only for the growth rate of the unstable modes, but also for the mode spectrum. The complex 3D structure can lead to strong coupling between the modes with different toroidal mode numbers $(n)$ which would modify the resulting stability of the plasma. The next step in this direction is the implementation of the real plasma environment (3D wall + feedback) into the codes together with a good model for the plasma-RWM interaction and a feedback algorithm. This is actually the subject of intensive research. The available theoretical works provide the basis for coupling the full 3D electromagnetic treatment of the wall with plasma calculations with linear MHD codes which includes influence of the plasma on the mode (e.g. ref.[41]). The first results demonstrating 
the feasibility of the codes coupling already exist [42] but further improvement of the codes is needed. Finally, such tools will be used to predict RWM stability in ITER and DEMO. One of the most crucial points in this modeling is the interaction of the RWM with the plasma which is taken into account in the linear MHD code. This is discussed in the next section.

\subsection{RWM interaction with plasma}

Experimental evidence for the importance of plasma - mode interactions was already obtained from the beginning of RWM investigations in tokamaks [11-13]. This evidence immediately posed questions about the physical mechanism behind the interaction and the possibility of implementing this interaction in linear MHD descriptions of the plasma. It was soon realized that Alfvén continuum damping alone is not sufficient to explain the experimental observations (e.g. a stable RWM region at very low plasma rotation as discussed later, etc.). The interaction is stronger than predicted by the continuum damping alone and more detailed analysis of the mode-plasma interaction has to be done. The physical mechanism which could provide this additional damping is an interaction of the mode (wave) with plasma particles. The main problem is to describe this interaction (energy transfer between RWM and plasma particles), which includes interplay with thermal particles as well as with fast particles. The difficulties arise when attempting to formulate a set of assumptions that enable the kinetic problem (wave-particle) to be reduced to an MHD formulation (fluid description). If such a description is found, it is possible to calculate the problem selfconsistently within the linear MHD approach. The simplest assumption which can be made is to represent this interaction as a fluid viscous force $\vec{\nabla} \cdot \vec{\Pi}_{1}$ in the linearized MHD momentum balance equation [5] (eq. 2):

$\rho_{0}\left(\gamma+i n \Omega_{0, p l}\right) \vec{v}_{1}=-\vec{\nabla} \cdot \vec{p}_{1}+\vec{j}_{1} \times \vec{b}_{0}+\vec{j}_{0} \times \vec{b}_{1}-\vec{\nabla} \cdot \vec{\Pi}_{1}-\rho_{0} \vec{U}\left(\vec{v}_{1}\right)$

where, $\rho$ is the density, $v$ is the fluid velocity, $b$ is the magnetic field, $j$ is the current, $p$ is the fluid pressure, $n$ is the toroidal mode number, $\gamma=\gamma_{0}+i \omega_{R W M}$ is the complex growth rate, and $\vec{U}=\vec{v}_{1} \times\left(\frac{\vec{v}_{0}}{R} \times \hat{\vec{R}}\right)+\left(\vec{v}_{1} \cdot \vec{\nabla}\right) \vec{v}_{0}$. Equilibrium quantities and perturbed quantities are denoted by subscript 0 and 1 , respectively. The rotation frequency of the plasma at the equilibrium $\Omega_{0, p l}$ is non-uniform and depends only on the equilibrium flux function.

The first model of this kind was proposed by Hammet and Perkins in 1990 [43]. It assumes an approximation for the Landau damping in the sound wave range. This model is 
often called the "sound wave damping" model. The force part has the following representation in this model:

$$
\vec{\nabla} \cdot \vec{\Pi}_{1}=\kappa_{\|} \sqrt{\pi}\left|k_{\|} v_{t h_{i}}\right| \rho_{0} \vec{v}_{1} \cdot \hat{b}_{1} \hat{b}_{1},
$$

here $k_{\|}=(n-m / q) / R$ is the parallel wave vector and $v_{t h_{i}}$ is the ion thermal velocity. The model contains a free "geometrical factor", $\kappa_{\|}$, which varies from 0.1 to 1.5 . A better model, developed by A. Bondeson and M.S.Chu a few years later, has no free parameters and takes into account mode coupling [44]. Thus, the "drag force" for a particular (m,n) mode depends on the toroidally coupled $(m \pm 1, n)$ modes and the modes are no longer independent.

Predictions using this model were in relatively good agreement with experimental measurements at that time, as obtained with the MARS-F code for the DIII-D case and shown in figure 6 [45].

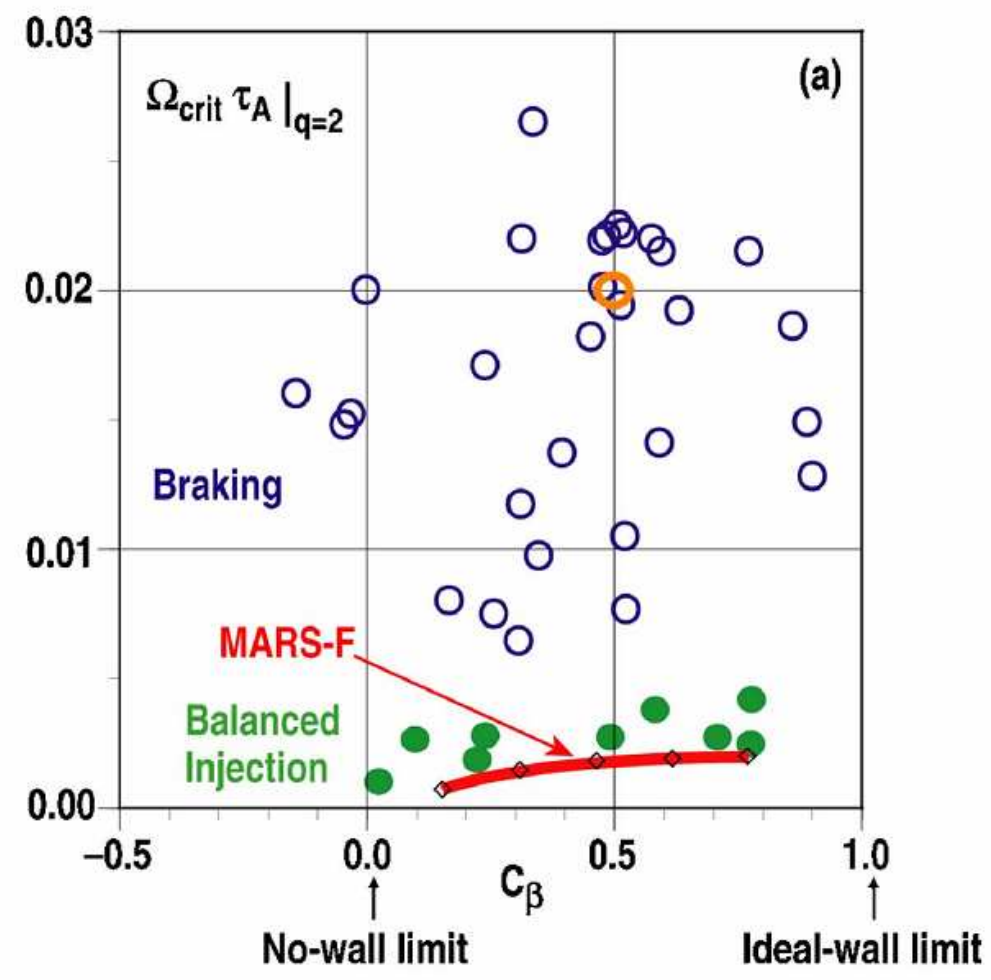

Figure 6. (color online) Comparison of the critical plasma rotation at $q=2$ resonant surface for RWM onset with MARS-F results (open diamonds and red curve) from Ref[45]. Experimental points for balanced NBI cases are shown in green (filled circles). Results of the braking ${ }^{2}$ experiments are shown in blue (open circles).

\footnotetext{
${ }^{2}$ Measurements of RWM stability in the presence of a strong neutral beam torque required some form of "magnetic braking" to reduce the plasma rotation to the critical value. Two braking methods were used: (1) reduction of the current in the error correction coils, allowing the uncorrected part of the intrinsic error field to create a drag on the plasma rotation; (2) application of an additional nonaxisymmetric field with an external set of coils.
} 
New problems come into play with reduction of the plasma rotation. It was clear from first estimations that plasma rotation in ITER will be significantly weaker than that in the present day devices since the relative input torque from Neutral Beam Injection will be smaller (taking into account the plasma volume in ITER). Thus, operations at low plasma rotation were identified as very important to make predictions for ITER. In order to address this issue, one neutral beam source was redirected in DIII-D to make balanced injection possible and to reduce the resulting torque on the plasma [45, 46]. JT-60U[47] and NSTX[48] were also operating in this regime and a strong reduction of the rotation threshold was observed as well. In spite of the relatively good agreement with the first results in DIII-D, it was soon realized that further reduction of the plasma rotation is possible, which does not agree with the Bondeson model predictions shown in figure 6. Here, the plasma rotation frequency required for the RWM stabilization is ploted versus normalized distance between the "no wall" and "ideal wall" limits, $C_{\beta}=\left(\beta_{N}-\beta_{N}^{\text {no-wall }}\right) /\left(\beta_{N}^{\text {ideal-wall }}-\beta_{N}^{\text {no-wall }}\right)$. A stable operations are not possible below critical rotation value which is in contradiction to stable operation at very low rotation. Thus, some assumptions of the Bondeson model are no longer valid in these low rotation plasmas and additional stabilization mechanisms have to be taken into account. In the model, several low frequency resonances were assumed to be zero, in particular the magnetic drift frequency and the diamagnetic drift frequency. To understand the problem including these resonances, it is useful to discuss another approach for investigation of plasma-RWM interaction. Up to now, only so called "self-consistent" MHD calculations have been discussed. In these calculations the eigenfunction is changed "self-consistently" according to linear MHD equations, but the interaction with the plasma is calculated with several assumptions. Another way to address the same problem is the so called "perturbative" approach.

The growth rate of the mode, $\gamma$, can be obtained from the perturbed MHD energy. In the RWM case we obtain the following expression [49]:

$$
\gamma \tau_{w} \simeq-\frac{\delta W_{M H D}^{\infty}}{\delta W_{M H D}^{b}},
$$

where, $\delta W_{M H D}^{\infty}$ and $\delta W_{M H D}^{b}$ are the fluid potential energy with a conducting wall at $\infty$ and $b$, respectively. The fluid energy includes the plasma and vacuum contributions. This formulation can be extended to include also the influence of the kinetic effects on the mode stability. The stability of the RWM is then determined by a dispersion relation derived from the kinetic-MHD energy principle for low frequency modes [50, 51]. 


$$
\gamma \tau_{w} \simeq-\frac{\delta W_{M H D}^{\infty}+\delta W_{k}}{\delta W_{M H D}^{b}+\delta W_{k}}
$$

where $\delta W_{k}$ is the kinetic part of the potential energy. In this approach, kinetic codes use fixed eigenfunctions from linear MHD to evaluate the interaction between the plasma and the mode for a given particle distribution (in another words to obtain $\delta W_{k}$ ).

The "perturbative" approach differs from the "self-consistent" approach in the following aspects:

(1) Kinetic modifications of the RWM structure are not taken into account. The mode is assumed to be "rigid", which means that the shape of the mode eigenfunction is not affected by the plasma.

(2) Kinetic effects are calculated accurately by evaluating kinetic integrals.

(3) The fluid continuum damping (due to the mode resonance with the Alfven waves) is not present in the perturbative calculations.

A comparison of the models is presented also in table 1, where advantages and disadvantages of the approaches are marked with “+”" and “_-“, respectively.

\begin{tabular}{|c|c|}
\hline $\begin{array}{l}\text { «Self-consistent» approach } \\
\text { (codes: MARS-K }{ }^{3}, \text { CASTOR-FLOW, etc) }\end{array}$ & $\begin{array}{l}\text { «Perturbative » approach } \\
\text { (codes: HAGIS, MISK, MARS-K }{ }^{3} \text {, etc.) }\end{array}$ \\
\hline System of linear MHD equations is solved & Kinetic integrals are calculated \\
\hline (-) Approximation for kinetic effects & (+) Correct calculation of the kinetic effects \\
\hline (+) Plasma influences on the mode structure & (-) The mode structure is fixed \\
\hline $\begin{array}{l}\text { (+) Interactions with Alfven continuum are } \\
\text { present }\end{array}$ & $\begin{array}{l}\text { (-) No interactions with Alfven and sound } \\
\text { wave continuum are present }\end{array}$ \\
\hline
\end{tabular}

Table 1. Comparison of the "self-consistent" and "perturbative" approaches. Advantages and disadvantages are marked by "“”” and "_.“, respectively.

Note that this kinetic-MHD energy principle treats the bulk plasma as an ideal one. Therefore, it was implicitly assumed that no island can develop at the rational surfaces $q=m / n$. The second point is that equations 4 and 5 allow an accurate evaluation of the RWM growth rate only near the point of marginal stability of the plasma. In spite of all the restrictions, very useful results can be obtained from the "perturbative" approach, which clarifies the plasmamode interaction. In what follows we discuss two questions to understand the physics of the resistive wall mode:

(1) Which resonances/particles impact strongly the mode stability?

\footnotetext{
${ }^{3}$ Both perturbative and self-consistent approaches are realized in the kinetic-MHD code MARS-K [52].
} 
(2) Where do the plasma-mode interactions mainly occur?

Qualitative answers to the two questions can be obtained from the kinetic approach.

Formally, $\delta W_{k}$ is a change of the potential energy which can be calculated for the kink mode as follows [53]:

$$
\delta W_{k}=\frac{1}{2} \int d \Gamma\left(m v_{\|}^{2}+\mu B\right) \delta f \sum_{m} \vec{\kappa} \cdot \vec{\xi}^{(m)^{*}}(r, t) e^{-i(n \phi-m \theta)}
$$

Here, $\phi$ is the toroidal angle, $\theta$ is the poloidal angle, $\vec{\kappa}$ is the curvature vector $\left(\vec{\kappa}=\vec{b} \cdot \nabla \vec{b}, \vec{b}=\vec{B} / B\right.$ ), and $\delta f=f-f_{0}$ is the perturbed part of the distribution function in the phase space volume element $\left(\Gamma=d^{3} x d^{3} v\right)$. This integral can be simplified for circular plasma in the large aspect ratio approximation. It is possible to show analytically that the resulting expression for $\delta W_{k}$ contains resonant conditions:

$$
\delta W_{k} \sim \sum_{l=-\infty}^{\infty} \frac{\left(\omega_{R W M}+i \gamma_{0}-n \omega_{E \times B}\right) \frac{\partial f_{j}}{\partial \varepsilon}-\frac{1}{e Z_{j}} \frac{\partial f_{j}}{\partial \Psi}}{\left\langle\omega_{D}^{j}\right\rangle+l \omega_{b}^{j}-i \nu_{e f f}^{j}+n \omega_{E \times B}-\omega_{R W M}-i \gamma_{0}}
$$

Here, $f_{j}$ is the distribution function of the particles $j, \varepsilon$ is the pitch angle, $\Psi$ is the magnetic flux, $Z_{j}$ is the effective charge, $v_{e f f}^{j}$ is the collision frequency. ${ }^{4}$ The first four frequencies in the denominator are the precession drift, bounce, collision, and $E \times B$ frequencies, respectively. The $E \times B$ frequency is $\omega_{E \times B}=\omega_{\phi}-\omega_{*_{i}}$, where $\omega_{\phi}$ is the toroidal rotation frequency and $\omega_{* i}$ is the ion diamagnetic frequency. For thermal particles the Doppler shifted mode frequency, $\omega_{E \times B}-\omega_{R W M}$, can resonate with the precession drift frequency $\left\langle\omega_{D}\right\rangle$ or with the bounce frequency, $\omega_{b}$, leading to large and complex $\delta W_{k}^{5}$. The discussed resonances can occur with the following frequencies:

- The transit frequency of the passing particles, $\omega_{t} \sim v_{t h} / R$;

- The bounce frequency of the trapped particles, $\omega_{b} \sim \sqrt{r / R}\left(v_{t h} / R\right)$;

- The precession drift motion, $\omega_{d} \sim \rho / r\left(v_{t h} / R\right)$;

Here, $\rho=m v_{\perp} / e B$ is the Larmor radius and $v_{t h}$ is the thermal velocity. These frequencies are quite different $\omega_{d}<<\omega_{b}<\omega_{t}$. Thus, a scan of the plasma rotation should identify several

\footnotetext{
${ }^{4}$ In practice, only first bounce harmonics, $l$, are important. For $l>|4|$ the resulting integral provides negligible changes of $\delta W_{k}$.

${ }^{5}$ The discussed expression is derived for trapped particles, but a similar approach could be applied to passing particles.
} 
resonances. A scan of $\delta W_{k}$ as a function of plasma rotation as calculated for a JET discharge is shown in figure 7 [17]. The thermal particle distribution function is considered to be isotropic with respect to pitch angle and Maxwellian with respect to energy. One can see significant changes in the kinetic part of the potential energy with changes of the plasma rotation. An increase of the $\delta W_{k}$ corresponds to an increase of the mode stability. Such an increase is seen close to the precession drift frequency and bounce frequency where the mode resonates with the motion of the particles.

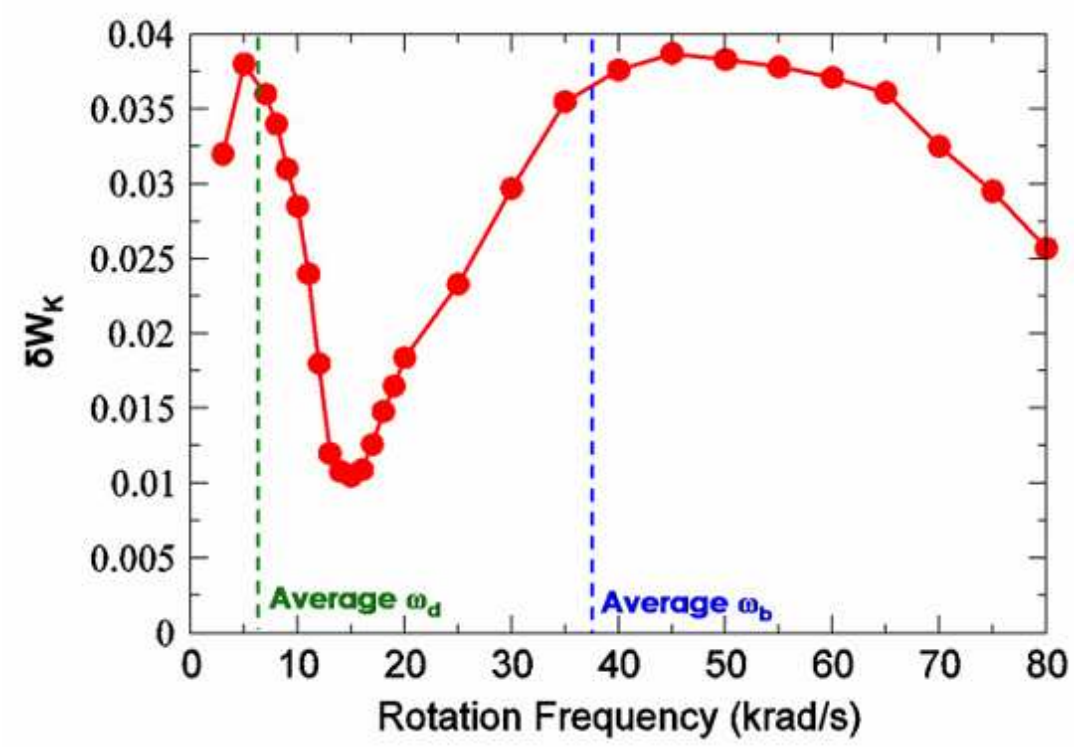

Figure 7. (color online) The real part of the change in the potential energy of the kink mode as a function of the plasma toroidal rotation. The influence of the kinetic damping changes significantly as the rotation varies. The figure is from reference [17].

Using the populations of marker particles, which are evenly distributed in the phase space, it is possible to identify the place of dominant plasma - mode interaction with the kinetic code [17]. Whilst the eigenfunction is large near the edge, calculations show that the strongest interaction is at mid-radius. It should be noted that in these calculations, a particle is considered as lost, when its orbit reaches the separatrix. This means that the role of particles near the plasma edge may be underestimated. The influence of faster particles could also modify the result, but the importance of the internal part of the eigenfunction is clear.

It has been shown that the low-frequency RWM can resonate with both the precession drift frequency and bounce frequencies of thermal ions (depending on the actual distribution of plasma particles). Thus, the assumption in the Bondeson model [44] is not valid anymore and these resonances have to be treated carefully. A recently developed model by Liu et. al. [54] takes into account all of these resonances. The magnetic drift frequency and diamagnetic drift frequency are no longer zero. The model treats the problem in full toroidal geometry 
where the kinetic integrals are evaluated. The kinetic effects are introduced into the MHD equations in a different way as compared to equations 2 . The equation now is written in the following form [52]:

$$
\begin{aligned}
& \rho_{0}\left(\gamma+i n \Omega_{0, p l}\right) \vec{v}_{1}=-\vec{\nabla} \cdot \vec{p}+\vec{j}_{1} \times \vec{b}_{0}+\vec{j}_{0} \times \vec{b}_{1}-\rho_{0}\left[2 \Omega_{0, p l} \hat{\vec{Z}} \times \vec{v}_{1}+\left(\vec{v}_{1} \cdot \nabla \Omega_{0, p l}\right) R^{2} \nabla \phi\right] \\
& \vec{p}=p \vec{I}+p_{\|} \hat{\vec{b}} \hat{\vec{b}}_{1}+p_{\perp}(\vec{I}-\hat{\vec{b}} \hat{\vec{b}})
\end{aligned}
$$

Here $\vec{v}, \vec{b}, \vec{j}, \vec{p}$ represent the velocity, magnetic field, current, and pressure tensor respectively, where $\hat{\vec{b}}_{1}$ and $b_{0}$ are defined as $\hat{\vec{b}}_{1}=\vec{b}_{1} / b_{0}, b_{0}=\left|\vec{b}_{0}\right|$. Equilibrium quantities and perturbed quantities are denoted by subscript 0 and 1 , respectively. $\hat{\vec{Z}}$ is the unit vector in the vertical direction, and $\vec{I}$ is the unit tensor. $\Omega_{0, p l}$ is the plasma rotation along the toroidal angle $\phi$. The kinetic effects enter into the equations via the perturbed kinetic pressure tensor $\vec{p}$. The full pressure tensor is self-consistently included into the MHD formulation via the momentum equation 8 . The parallel and perpendicular components of the pressure tensor are calculated by integration over the particle velocity space.

As was pointed out, any inclusion of the kinetic effects into the MHD description requires a set of approximations which will work only if all of the important physical effects are taken into account. In spite of the fact that the Liu model include a much more careful treatment of the problem, some assumptions were made. It neglects the perturbed electrostatic potential, assumes zero banana width for trapped particles and no finite Lamoure radius (FLR) corrections to the particle orbits. These effects should not be important for the RWM (but could be crucial for other MHD instabilities). On the other hand, there is no guarantee that all of the important effects are present, especially if one keeps in mind the history of the model development. At the same time, "perturbative" calculations with kinetic codes could be extremely useful for addressing the same problem from the other side. Although it is not possible now to benchmark the two approaches, one could get an idea about the possible influence of the plasma on the mode from the "perturbative approach" and about changes of the eigenfunction from "self-consistent" calculations.

Thus, the most natural way is to merge the two approaches in the future. Such merging could be done, for example, by extending kinetic codes to self-consistent calculations. These calculations will be probably very time consuming, but they are necessary to benchmark the plasma-RWM interaction model at least in several cases. This model finally will be included 
into a linear MHD code which will be coupled to 3D external structures, as discussed in section 5.1.

At present, simultaneous calculations with the "perturbative" and "self-consistent" approaches is the best way to address the RWM stability problem. In this way estimates of kinetic effects and changes of the eigenfunction due to interaction with the plasma can be obtained. Results of such calculations for ITER are discussed in section 6 .

\subsection{Experimental evidence of kinetic effects on RWM stability}

The complex problem of the interaction between plasma particles and the RWM was discussed in the previous section. The RWM stability is a comprehensive quantity which encompasses many effects. It is a challenging task to separate the kinetic effects in experiments and to measure them. In spite of these difficulties, some measurements show the importance of the kinetic effects. As discussed before, the stability of the RWM should vary with changes in the plasma rotation. The active "MHD spectroscopy" technique could be used to characterize the stability of the RWM. In these experiments the plasma response to externally applied quasistatic $n=1$ magnetic fields was measured in DIII-D for various plasma rotations [55]. The main result was that the changes in the RWM stability depend on the plasma rotation and are in qualitative agreement with predictions of the kinetic calculations. The features at low rotation seem to be directly related to the resonance with the precession frequency of the thermally trapped ions. This effect is expected from kinetic calculations.

Analysis of the plasma stability in NSTX with and without feedback at low plasma rotation also points out the importance of kinetic effects since these effects could explain the observed variation of the RWM stability with evolution of the rotation profile [56].

These results are the first experimental evidence for the importance of kinetic effects. The results are promising, but further investigations are required to prove the impact of kinetic effects in experiments and ideally to measure the actual magnitudes of these effects.

\section{RWM stability in ITER}

The ITER project $[57,58,59]$ will be one of the most important experiments in fusion research for decades to come. Thus, accurate predictions for ITER operation in advanced scenarios are of particular interest. The determination of the RWM stability boundary in ITER now, and verification of that boundary during ITER operation are necessary points on the way 
to DEMO, which will be the first prototype fusion energy reactor. Accurate evaluation of the stability boundary will be especially important for advanced tokamak operation in DEMO [60-63]. In this case, these predictions will influence strongly even the size of the device. In the next two sections the influence of the thermal and fast particles in ITER on RWM stability are discussed. The new model [54] was used to study RWM stability in ITER together with perturbative calculations. Both types of calculations were carried out with the MARS-K code.

\subsection{Influence of the plasma rotation on the RWM stability}

Investigations on the stabilization effects of precession resonances at slow rotation velocities in ITER was present in Ref [64]. The calculations were based on the steady state 9MA scenario in ITER with high pressure and high bootstrap current fraction, $\beta_{N, n o-\text { wall }} \approx 2.7, \beta_{N, \text { ideal-wall }} \approx 3.8$ (scenario 4 ) [65]. The predicted rotation at the $\mathrm{q}=3$ surface is less than $0.25 \% \omega_{\text {Alfvén }}$ [64]. (The $\mathrm{q}=2$ surface, which is typically used for critical rotation value (e.g. figure 6), is absent for these ITER plasmas.) The predicted plasma central rotation, $\omega_{0}$, is less than $2 \% \omega_{\text {Alfvén }}$. One has to note that there is significant uncertainty for the predicted rotation amplitude and the rotation profile for ITER. The presented values give the upper boundary for the rotation in ITER and the real values could be much smaller.

For steady-state advanced scenarios, the self-consistent calculations predict a full stabilization of the RWM at very slow plasma rotation (less than $0.2 \%$ of the Alfvén speed at the plasma centre) and moderately high plasma pressures

$\left(C_{\beta}=\left(\beta_{N}-\beta_{N}^{\text {no-wall }}\right) /\left(\beta_{N}^{\text {ideal-wall }}-\beta_{N}^{\text {no-wall }}\right)=0.4\right)$. It is important that no slow rotation threshold, below which the mode becomes unstable, was found in the calculations (see figure 8 , right). Instead, an upper stability threshold was found. This contradicts the previously discussed Bondeson model predictions, in which a slow rotation threshold is present, see figure 6. Existence of an upper threshold could be due to the inclusion of the precessional resonances in the simulations. Actually, further increase of plasma rotation could again stabilize the mode above the upper limit due to resonance damping, continuum damping, and inclusion of fast particle effects or any combination of these processes. As for ITER, the results show the mode to be stable well above the "no wall" limit.

Even more optimistic results were obtained by the "perturbative" approach, where the eigenfunction of the ideal kink mode (from fluid RWM calculations) is used to evaluate the kinetic integrals (see figure 8 , left). The difference in the results of the perturbative and non- 
perturbative calculations is partially due to the modification of the RWM eigenfunction, but primarily due to the self-consistent determination of the mode eigenvalue in the latter approach as discussed in the previous sections.

In summary, for a plasma toroidal rotation speed up to the predicted value for ITER, the kinetic damping of the RWM is mainly provided by precessional drift resonances of trapped thermal particles. This damping stabilizes RWM up to $\beta_{N}$ values half way between the "no wall" and "ideal wall" limits $\left(C_{\beta}=0.4\right)$.
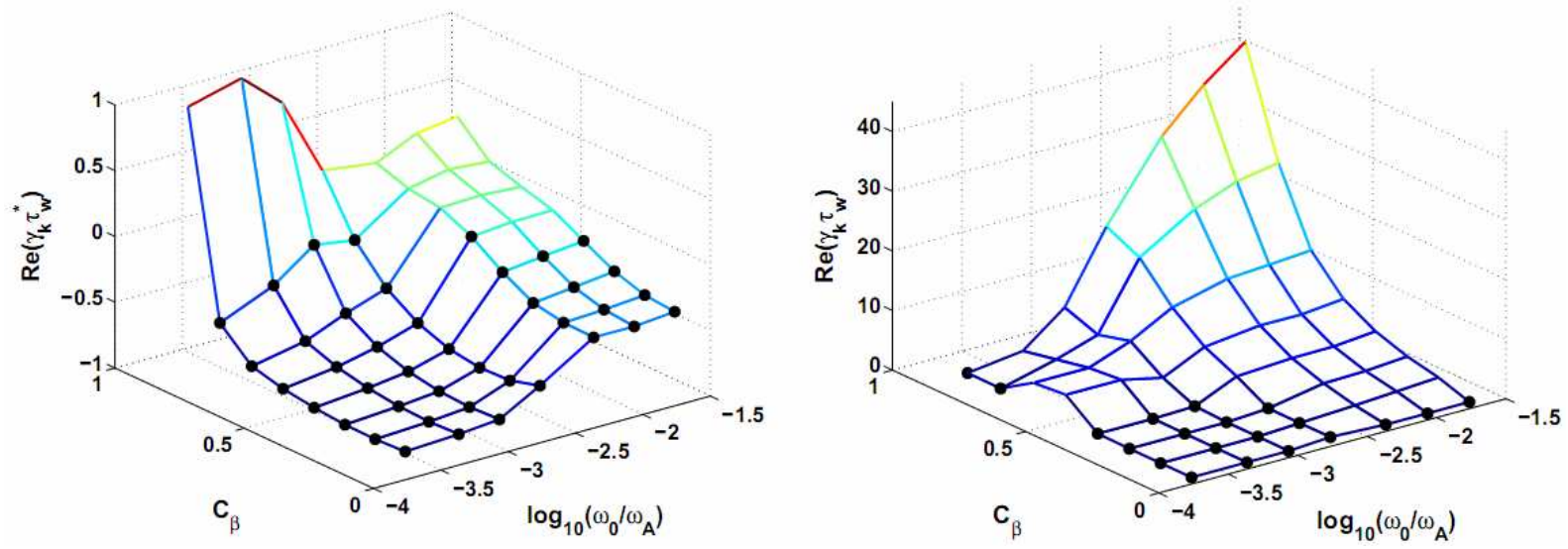

Figure 8. Growth/damping rate of the RWM for ITER advanced tokamak plasmas, predicted by perturbative kinetic calculations (left) and self-consistent kinetic calculations (right). The precessional resonance damping is included. Black dots indicate a stable RWM. Figures are from Ref.[66].

\subsection{Influence of the alpha particles on RWM stability}

One of the main differences between ITER plasmas and the plasmas in present day tokamaks is the existence of a large fraction of fast alpha particles resulting from fusion reactions. To understand the whole picture in ITER one has to take into account the influence of these particles on the RWM stability. Such modeling was published in the recent paper by Y. Liu [67]. The calculations were made based on the same steady state scenario as in the previous section 6.1 . The $\alpha$ contribution is found to be generally stabilizing, compared with the thermal particle kinetic contribution alone (which was discussed before). This conclusion is based on the two approaches. The "perturbative" approach generally predicts higher stabilization than the "self-consistent" approach which is a typical result for such comparisons. The stabilizing effect from $\alpha$ particles is more pronounced at fast enough plasma rotation, when the rotation frequency matches roughly the $\alpha$ precession frequency, which is of the order of a few percent of the toroidal Alfven frequency for ITER. Thus, this stabilization is expected to be significant only if the central plasma rotation will be close to predicted upper level in ITER or higher. One has to note that the discussed modeling neglects the effect of the 
finite drift orbit widths of trapped $\alpha$ particles, which is expected to be reasonably large compared with the plasma minor radius in ITER. Thus, further investigations are needed in this area to clarify the effect of the $\alpha$ particles.

\section{Triggering of RWM at low plasma rotation}

There is one important point which has to be mentioned together with RWM physics in order to complete the RWM physics discussion. It is not directly responsible for the physics of the RWM, but determines the stability of the discharge with respect to the RWM. Several years ago, it was shown that the RWM could be stabilized at low plasma rotation [46, 47]. However, stable operation without RWM in slowly rotating plasmas is not guaranteed [68]. Various MHD activities can trigger the RWM near the no-wall limit. These include: (i) Edge Localized Modes (ELMs), (ii) off-axis fishbones, and (iii) the energetic particle-driven wall mode (EWM) [69]. It was shown recently that the latter two modes have the same origin [70].

A global MHD event can interact with the RWM branch and can trigger the RWM if the following conditions are fulfilled:

- The MHD event produces distortions in the same place where the potential RWM could be present.

- The MHD event has a component with the same toroidal mode number $n$ (typically $n=1$ for $n=1$ RWM).

- The MHD event and the potential RWM have comparable frequencies.

This forced excitation of RWM takes place within a fraction of the time scale of the driving MHD event which is typically much faster than the wall time constant. It is interesting that the magnitude ratio between the exited mode and the driving force is similar in all these cases. This emphasizes similarities for the triggering procedure.

The existence of the different trigger mechanisms gives additional confirmation of the global nature of the RWM eigenfunction (see section 4). In case of an ELM triggering, external part of RWM is coupled to an ELM perturbation which has components only at the plasma edge. Fishbone-driven RWMs are triggered differently. The energetic ions injected by neutral beams are expelled by the off-axis fishbone bursts with maximal perturbation at the middle of the plasma radius $(\rho \approx 0.5)$. Thus, the coupling occurs between the off-axis fishbone and internal part of the RWM eigenfunction.

The existence of these coupling phenomena move the problem of RWM stability and control from a single mode consideration into the complex space of the global MHD stability 
control in the plasma. This is, in some sense, similar to the challenges which one faces in trying to sustain the advanced scenario in a tokamak. As it was shown by Luce [71] one has to meet simultaneously the correct point in current drive, transport, and MHD stability, which requires integrated control for advanced scenario regimes. The same is true for control of RWM in this regime. It has to be integrated into the global MHD control approach to avoid the RWM triggering or suppress triggered mode by proper feedback action. It has to be mentioned that also resistive instabilities can be destabilized with increase of $\beta_{N}$ [72]. A possible solution is to avoid the most dangerous rational surfaces or stabilize the modes with external current drive.

\section{Conclusions and discussion}

The advanced tokamak regime is a promising candidate for steady state tokamak operation which is preferable for a fusion reactor. This regime is fully non-inductive and the plasma current is sustained by the bootstrap current. The bootstrap current value is proportional to the pressure gradient. Thus, high pressure is unavoidable in the advanced regime. The resistive wall mode is a global kink instability which appears in tokamaks at high pressure. It has typically a single toroidal mode number $(n=1)$ and multiple poloidal mode numbers $(m=2 . .6)$. Due to its global nature, RWMs interact with plasma particles, and resistive walls and can be triggered by several other instabilities. The electromagnetic part of the resistive wall mode interaction is relatively well understood. The interaction of the resistive wall mode with the plasma is a more challenging problem and is still open. Currently, there are two approaches on how to address this interaction: the "self-consistent" and the "perturbative" methods. The first approach contains an approximation for the kinetic effects, but calculates RWM stability self-consistently. The second approach treats the kinetic interaction correctly, but does not treat the problem self-consistently. These two approaches are complimentary and have to be used simultaneously. For steady-state advanced scenarios in ITER, the self-consistent calculations predict a full stabilization of the RWM at very slow

plasma rotation and moderately high plasma pressures $\left(C_{\beta}=0.4\right)$. "Perturbative" calculations provide even higher stabilization. Both results are very positive for ITER and show that a substantial increase in fusion performance is possible even without active feedback control. At the same time, a self-consistent modeling with a correct kinetic description is required to make correct predictions. Even if such a modeling requires large computer resources, one has to perform it to verify approximate models for linear MHD codes. This verification has to be 
done also experimentally. Absence of a verified model for linear MHD is one of the main unknowns in RWM physics presently. A linear MHD code with a verified model could be coupled with an electromagnetic code (3D external vessel + feedback coils + error fields + feedback control) to model the RWM in a realistic environment. The first example of such a coupling already exists, which shows feasibility of the task. This set of the codes allows us to predict the spectrum and growth rates of the RWM in future machines such as ITER and DEMO.

The other important point is the development of integrated scenario control for advanced tokamak operations. This development includes integrated control and avoidance of RWMs and other MHD instabilities, which could act as a trigger. One of the possible routes is to avoid RWM triggering by flexible feedback correction with a wide bandwidth feedback system. In this scheme, the feedback corrects slow (error field correction) and fast (RWM) dynamics simultaneously [73]. A possible variant of an integrated control strategy is the "three step strategy":

1. Avoid RWM if possible. This implies correction of error fields and mitigation of ELMs. (Only relatively small perturbations from other instabilities are allowed).

2. Prevent coupling to the wall and suppress small RWM by active feedback if (1) fails.

3. Decouple from the wall, rotate and suppress by feedback if (1) and (2) fail.

We define the proposed strategy in such a way that the efforts are minimal at the beginning. The second step (and especially the last one) are more challenging and should be done only if it is absolutely necessary. This will keep the amount of possible actions and efforts at the minimal level. One can see that this strategy has to be integrated into an overall MHD control. It is also clear that some points have to be addressed experimentally (for example decoupling of RWM from the wall is demonstrated for RFPs [33-35] but has to be tested for tokamaks).

The other important issue is the stability boundaries $\beta_{N, n o-\text { wall }}$ and $\beta_{N, \text { ideal-wall }}$. There are three important points which one has to keep in mind when working with these terms.

1) The stability boundary is different for different mode numbers (see figure 1).

2) These two limits were defined on the basis of an ideal MHD approximation. Kinetic effects lead to changes in the mode stability and can considerably change the stability boundaries as was shown in the previous sections (see figure 8). In other words, it is not possible to keep the plasma stable in the presence of an ideal instability. This ideal instability limit is a hard limit for the plasma confinement. At the same time, stability boundaries for the RWM are strongly affected by kinetic effects. 
3) $\beta_{N}$ represents the average pressure in the plasma. The real drive for the mode comes from the pressure profile gradient, not from the pressure itself. Also, an increase of the gradients is unavoidable for a strong increase of the total plasma pressure. Total pressure $\beta_{N}$ can be moderately increased without increasing the gradient in the mode region. Thus, different stability situations are possible for the same $\beta_{N}$ even without inclusion of the kinetic effects.

Identification of the "true" stability boundary requires modeling with accurate profile gradients and an accurate account of the particle influence. Such modeling is challenging because profile measurements tend to be of insufficient quality (e.g. current profile, rotation profile, etc), and because of the lack of a "final" model for RWM-plasma interaction. Thus, rough estimates in terms of well measured quantities, such as $\beta_{N}$, are required to understand the position of the plasma in operational space and to compare the results from different experiments. At present, the experimental identification of the RWM stability boundary is relatively simple. One of the methods is to study the plasma response to external perturbations. The reaction of the plasma increases after crossing the "no wall" limit. The typical estimate for the "ideal wall" limit is a factor of two above the "no wall" limit. But this is a rule of thumb which could be wrong under a certain circumstances. The value of $\beta_{N}$ is the best indicator available but the factors affecting the stability limits have to be kept in mind.

The next important issue is the mode "rigidity". This term is defined differently by different authors as discussed in section 4. In many cases, people assume a "rigid" mode structure using one of these definitions. At the same time, there is also evidence for "nonrigidity" of the mode (for example in ref.[48]). The question of the mode "rigidity" has to be addressed carefully in future experiments because "non-rigidity" could change requirements for the mode stabilization and for the design of the RWM feedback control. In this situation, experiments on RFPs could be of a particular interest. The large number of control coils allow to vary the configuration in a very flexible way and to explore the mode rigidity in terms of local toroidal/poloidal suppression as well as in terms of unstable modes with different toroidal mode numbers $[19,74,75,76]$.

We would like to note that RWM physics could be important for a future power plant. This power plant has to be robust and stable in its operation window. At the same time, its operation space does not necessarily need to be as wide as in modern tokamaks or as in ITER. One has to decide in which scenario the plant will operate before it will be build. The cost of 
the electricity becomes also one of the major criteria for such power plant. The cost could be reduced by:

- reduction of the reactor size, which leads to more advanced tokamak operations (with higher bootstrap current fraction),

- use of a minimal set of diagnostic and control tools,

- operation at the maximum achievable normalised beta $\left(\operatorname{costs} \propto \beta_{N}^{-0.4}\right)$ [77],

It is also clear that the power plants will be bigger than ITER [63]. Thus, the plasma rotation is expected to be much smaller compare to ITER which could open a stable operation space due to resonance of the particles with the RWMs. Thus, it could be desirable to design the future power plant for the operation space where no RWM control is required and high $\beta_{N}$ values are achieved by kinetic stabilization of RWMs. In this case no special control coils are required which will strongly simplify the device and reduce the costs. This shows the importance of the problems discussed in this paper, and, in particular, for a correct model for the plasma-RWM interaction. A feasibility study for such a scenario requires a lot of work both for the sustainment of the required plasma profiles and for a quantitative understanding of the kinetic effects in RWM physics. It is clear that, presently, the power plant issues are much more uncertain than for ITER and thus further investigations will be required.

Finally, the recent review of M.S.Chu and M. Okabayashi [3] is recommended as a nice and detailed survey of RWM stabilization technique.

\section{Acknowledgments}

The author would like to thank H. Zohm, S. Gunter and E. Strumberger for their contribution and many useful discussions about the resistive wall mode physics. The author is indebted to H. Zohm, D. Yadikin, O. Dumbrajs and R. McDermott for their careful reading of this manuscript and for their constructive remarks and suggestions. The author would like to thank V. Pustovitov and Y. Liu for very helpful and fruitful discussions at conferences and meetings. The author would like to thank D. Yadikin, T. Boltzonella, S C Guo, M. Baruzzo and the RFX-mod team for their help in experiments on RFX-mod.

\section{References}

[1] N. J. Fisch, Rev. Mod. Phys. 59, p.175 (1987)

[2] A. G. Peeters Plasma Phys. Control. Fusion 42, p.B231 (2000)

[3] M.S. Chu and M. Okabayashi, Plasma Phys. Control. Fusion 52, 123001 (2010) 
[4] A. Bondeson and D.J.Ward, Phys. Rev. Let. 72, p.2709 (1994)

[5] J. Manickam et.al., Phys. Plasmas 1, p.1601 (1994)

[6] A. M. Garofalo, Nuclear Fusion, Vol. 40, No. 8, 1491 (2000)

[7] E. J. Strait, et.al. in Fusion Energy 2002 (Proc. 19th Int. Conf. Lyon, 2002) (Vienna: IAEA) CD-ROM file EX/S2-1 and http://www-pub.iaea.org/MTCD/publications/PDF/csp_019c/html/node137.htm

[8] H. Reimerdes, Phys. Plasmas 13, 056107 (2006)

[9] M. S. Chu et. al. Phys. Plasmas 2 (6), p.2236 (1995)

[10] R. Betti and J. P. Freidberg Phys. Rev. Lett. 74, p.2949 (1995)

[11] A. M. Garofalo et.al. , Phys. Rev. Lett. 82, p.3811 (1999)

[12] A. M. Garofalo et.al. , Phys. Plasmas 10, p.4776, (2003)

[13] M. Okabayashi, Plasma Phys. Control. Fusion 44, p.B339-B355 (2002)

[14] A. M. Garofalo et.al., Nucl. Fusion 42, p.1335 (2002)

[15] S. Sabbagh et.al., Nucl. Fusion 46, p.635 (2006)

[16] M. Okabayashi, Nucl. Fusion 49, 125003 (2009)

[17] I. T. Chapman et.al., Plasma Phys. Control. Fusion 51, 055015 (2009)

[18] Yueqiang Liu, Plasma Phys. Control. Fusion 51, 115006 (2009)

[19] M. Takechi, et.al., 37th EPS Conference on Plasma Physics, Dublin, Ireland, 21 - 25

June, 2010,P2.192, http://ocs.ciemat.es/EPS2010PAP/html/author.html

[20] V. D. Pustovitov, Plasma Phys. Control. Fusion 48, 51 (2006)

[21] V. D. Pustovitov, Plasma Phys. Rep., Vol. 30, No. 3, p.187 (2004)

[22] A. H. Boozer, Phys. Rev. Let. 86, p5059, (2001)

[23] M. Okabayshi, N. Pomphrey, R.E. Hatcher, Nucl. Fusion, Vol. 38, 1607 (1998)

[24] J. M. Finn , Phys. Plasmas 2 , p. 198 (1995)

[25] R. Fitzpatrick Phys. Plasmas, Vol. 9, No. 8, p. 3459 (2002)

[26] S.C.Guo Phys. Plasmas, Vol. 6, No. 10, October p. 3868 (1999)

[27] D. Yadykin, et.al., Plasma Phys. Control. Fusion 53, 085024, (2011)

[28] M. Okabayashi, et.al., 36th EPS Conference on Plasma Physics, Sofia, Bulgaria, June 29July 3, 2009, ECA Vol.33E, P-4.141 (2009) or CD-ROM file P4.141 http://epsppd.epfl.ch/Sofia/start.htm

[29] Y. Liu, et.al., Phys. Plasmas 17, 072510 (2010)

[30] P. R. Brunsell et al., Phys. Rev. Lett. 93, 225001 (2004)

[31] P. R. Brunsell et al., Plasma Phys. Control. Fusion 47, B25 (2005)

[32] T. Bolzonella, Fusion Engineering and Design 82 p.1064-1072 (2007)

[33] V. Igochine, T. Bolzonella, S. C. Guo, et al., in Proceedings of the 35th EPS Conference 
on Plasma Physics, Hersonissos, 2008, ECA 32D, P2.066 and http://epsppd.epfl.ch/Hersonissos/html/i_index.htm

[34] T. Bolzonella, V. Igochine, S. C. Guo, et al., Phys. Rev.Lett. 101, 165003 (2008).

[35] V. Igochine, T. Bolzonella, S. C. Guo, et al., Plasma. Phys. Control. Fusion 51, 055008 (2009)

[36] J.R. Drake et.al. in Fusion Energy 2008 (Proc. 22th Int. Conf. Geneva, Switzerland, 2008) (Vienna: IAEA) CD-ROM file EX/P9-7 and http://www-naweb.iaea.org/napc/physics/fec/fec2008/html/node499.htm

[37] F. Villone et.al., Phys. Rev. Lett. 100, 255005 (2008)

[38] V. D. Pustovitov, Plasma Phys. Rep., Vol. 37, No. 1, p. 35 (2011)

[39] E. Strumberger, et.al., Phys. Plasmas 15, 056110 (2008)

[40] F. Villone, et. al. 36th EPS Conference on Plasma Physics, Sofia, Bulgaria, June 29July 3, 2009, ECA Vol.33E, P4.166 (2009) or CD-ROM file P4.166 http://epsppd.epfl.ch/Sofia/start.htm

[41] V. D. Pustovitov, Plasma Phys. Control. Fusion 50, 105001 (2008)

[42] Y. Liu and F.Villone, Plasma Phys. Control. Fusion 51, 115008 (2009)

[43] G. W. Hammet and F. W. Perkins, Phys. Rev. Lett. 64, p.3019 (1990)

[44] A. Bondeson and M. S. Chu, Phys. Plasmas 3, p.3013 (1996)

[45] E. J. Strait et al., Phys. Plasmas 14, 056101 (2007)

[46] H. Reimerdes, et.al., Phys. Rev. Lett 98, 055001 (2007)

[47] M. Takechi, et. al., Phys. Rev. Lett 98, 055002 (2007)

[48] S.A. Sabbagh, et.al., Phys. Rev. Lett 97, 045004 (2006)

[49] S. W. Haney and J. P. Friedberg, Phys. Fluids B 1, p.1637 (1989)

[50] B. Hu and R. Betti, Phys. Rev. Lett. 93, 105002 (2004)

[51] B. Hu et.al., Phys. Plasmas 12, 057301 (2005)

[52] Y. Liu et.al., Physics of Plasmas 15, 112503, (2008)

[53] B. N. Breizman, J. Candy, F. Porcelli and H. Berk, Phys. Plasmas 5, p. 2326 (1998)

[54] Y. Liu et.al., Phys. Plasmas 15, 092505 (2008)

[55] H. Reimerdes et.al., Phys. Rev. Lett 106, 215002 (2011)

[56] S.A. Sabbagh et.al., Nucl. Fusion 50025020 (2010)

[57] M Shimada et al., Progress in the ITER Physics Basis Chapter 1:

Overview and summary 2007 Nucl. Fusion 47 S1

[58] T C Hender et al. Progress in the ITER Physics Basis Chapter 3: MHD 
stability, operational limits and disruptions 2007 Nucl. Fusion 47

S128-S202

[59] C. Gormezano et al., Progress in the ITER Physics Basis Chapter 6:

Steady State Operation 2007 Nucl. Fusion 47 S285

[60] J. Garcia et.al., Nucl. Fusion 48, 075007(2008)

[61] Stacey, W. M. Front Matter, in Fusion: An Introduction to the Physics and Technology of Magnetic Confinement Fusion, 2nd Edition, Wiley-VCH Verlag GmbH \& Co (2010)

[62] I.T. Chapman, et.al., Fusion Engin. and Design, 86, Issues 2-3, Pages 141-150 (2011)

[63] H. Zohm, Fusion Science and Technology, 58, p. 613 (2010)

[64] Y. Liu, Nucl. Fusion 49, 035004 (2009)

[65] Polevoi A. et all, in Fusion Energy 2002 (Proc. 19th Int. Conf. Lyon, 2002) (Vienna: IAEA) CD-ROM file and

http://www.iaea.org/programmes/ripc/physics/fec2002/html/fec2002.htm

[66] Y. Liu et all, in Fusion Energy 2010 (Proc. 23rd Int. Conf. Daejeon, 2010) (Vienna:

IAEA) CD-ROM file [TH/P9-26] and

http://www-naweb.iaea.org/napc/physics/FEC/FEC2010/html/index.htm

[67] Y. Liu , Nucl. Fusion 50, 095008 (2010)

[68] Okabayashi, et.al. Nucl. Fusion 49125003 (2009)

[69] G. Matsunaga, et.al., Phys. Rev. Lett, 103, 045001 (2009)

[70] M. Okabayashi, et.al. Phys. Plasmas 18, 056112 (2011)

[71] T.C. Luce, Phys. Plasmas 18, 030501 (2011)

[72] D. P. Brennan, et.al., Phys. Plasmas, Vol. 10, No. 5, May, p.1643 (2003)

[73] Y. In, et.al., Plasma Phys. Control. Fusion 52, 104004 (2010)

[74] T. Boltzonella, et.al., in Fusion Energy 2010 (Proc. 23rd Int. Conf. Daejeon, 2010)

(Vienna: IAEA) CD-ROM file [EXS/P5-01] and

http://www-pub.iaea.org/mtcd/meetings/cn180_papers.asp

[75] T. Boltzonella, et.al., 37th EPS Conference on Plasma Physics, Dublin, Ireland, 21 - 25

June, 2010, P2.176, http://ocs.ciemat.es/EPS2010PAP/htm1/author.html

[76] D. Yadikin, P. R. Brunsell and J. R. Drake, Plasma Phys. Control. Fusion 48, p.1 (2006)

[77] D. J. Ward et al. in Fusion Energy 2000 (Proc. 18th Int. Conf. Sorrento, 2000) (Vienna: IAEA) CD-ROM file FT/P2-20 and

http://www.iaea.org/programmes/ripc/physics/fec2000/html/node1.htm 